\title{
The Obstacles of Copyright Protection of Drug Instructions and Its Countermeasures*
}

\author{
Yuqing Zhang \\ College of Humanities \\ Hubei University of Chinese Medicine \\ Wuhan, China 430065
}

\begin{abstract}
With the rapid development of the pharmaceutical industry, intellectual property has become a new entry point for competition in the industry, and there has been an increase in the number of copyright infringement cases related to drug instructions. However, at present, China's attitude towards whether or not the drug instructions can be protected by the Copyright Law is unknown, leading to different judgments when trying such cases. This papers uses points out different viewpoints when trying infringements of drug instructions with two typical cases in China, discusses the obstacles and causes of copyright protection for drug instructions, and proposes several countermeasures to address these obstacles.
\end{abstract}

Keywords—drug instructions; originality; copyright

\section{INTRODUCTION}

With the rapid rise of the bio-pharmaceutical industry, the benefits of traditional competitive means are getting smaller and smaller, and the role of such newly created competitive methods such as intellectual property is becoming increasingly significant. Therefore, pharmaceutical companies begin to protect their own research results by applying for patents or keeping their experimental data confidential. In order to make longer-term profit of drugs, some pharmaceutical companies also use the means of protecting the copyright of drug instructions to enhance market competitiveness. Concerning the healthy development of the pharmaceutical industry and people's health, it is imperative to properly resolve the issue of copyright protection of drug instructions.

\section{THE DEFINITION OF DRUG INSTRUCTION}

\section{A. The Meaning of Drug Instruction}

The drug instruction is a statutory document that contains important information about the drug and is a statutory guide for selecting drugs. The instruction after approval of a new drug may not be modified without authorization. The contents of the drug instruction should include the drug's name, specifications, manufacturer, drug approval number, product lot number, expiration date, main ingredients, indications or major functions, usage, dosage, contraindications, adverse reactions, and precautions. Instruction for preparations of

* About the author: Yuqing Zhang, female (1977---), Lecturer of College of Humanities, Hubei University of Chinese Medicine, main research direction: civil and commercial law. traditional Chinese medicines should also include main medicine (component) traits, pharmacological effects, storage, and so on.

\section{B. Differences between Drug Instructions and General Instructions}

Drugs are special commodities and their instructions should be different from those of general commodities. Drug instructions can provide medication information, which is an important way for medical personnel and patients to understand the drugs. The standard degree of drug instructions is closely related to the quality of medical care. Therefore, the law strictly regulates the content and format of drug instructions. In addition, drug instructions should be revised in a timely manner based on the safety and effectiveness of the drug after coming into the market.

\section{DOMESTIC AND FOREIGN LEGISLATION AND PRACTICE}

\section{A. United States}

In the 1980s, the United States promulgated the Drug Price Competition and Patent-period Compensation Law, which mitigated the conflict of interest between generic drugs and original drug dealers. On the one hand, it encouraged original drug dealers to develop new drugs and also promoted the benign competition of generic drug industry. At the end of the 1990s, Smithkline Beecham Company sued Watson Company, for the former believed that the defendant's generic drugs used the same manual and video tape as their new drug. Although the court in the southern United States supported Smithkline Beecham's claims at beginning, it subsequently withdrew the prohibition of infringement against Watson Company. The court confirmed that Smithkline Beechem's drug use manuals and video tapes should be protected by the copyright law but the practice of generic drugs did not violate the relevant policies formulated by the FDA. Eventually, the US judicial practice community established the principle of trial application that "In case of conflict between Food Drugs and Cosmetic Act and the Copyright Law, the Food Drugs and Cosmetic Actshall prevail."

\section{B. Australia}

In the case that Sanofi-Aventis sued Apotex for drug infringement on drug instruction, the Australian Federal Court 
supported the viewpoint that instructions do not lose copyright due to normalization, and shew an implied license to Apotex's claim that generic drugs shall be allowed to use the instruction of the original drug for public interest. Under the influence of this case, the amendment to the Medical Supplies Act enacted by Australia on May 28, 2011 came into force, which provided that the provision, reproduction, publication, dissemination, modification of part or all of the drug instructions specified in Section 25AA of the Medical Supplies Act of 1989are behaviors of reasonable use, and do not constitute infringement to the copyright of the drug instruction. Compared with the Drug Price Competition and Patent-period Compensation Law of the United States, this Act provided too wide on content, entity, and scope of the reasonable use of the drug instruction, which may cause the reasonable use of the drug instruction to spread widely to book publishing and other commercial activities. There is no restriction on the entity of reasonable use, and the editors, publishers, and other industry entities can all use the drug instruction, which is obviously not conducive to the copyright protection of drug instructions.

\section{Taiwan, China}

The formulation the Guidelines for Drug Registration, Examination and Approval of Taiwan in China has drawn from the Drug Price Competition and Patent-period Compensation Law of the United States. The rules stipulate that generic drugs should be reviewed in accordance with the contents of the original drug'sinstruction when formulating the instructions, rather than that the generic drug's instruction should be translated in detail in accordance with the original drug's instructions. In Taiwan, three methods are mainly taken to resolve infringements in drug instruction: The first is not to directly indicate that drug instructionsare protected by copyright, the second is not to recognize the originality of the generic drug's instruction, and the third is to recognize the original drug's instruction is protected by copyright, but it relaxes the reproduction and translation of the generic drug's instruction. Either way, they do not recognize generic drug's copyright infringement.

\section{Chinese Mainland}

China's Regulations on Drug Instruction and Label Management and Drug Administration Law have clearly stipulated the content and format of drug instructions, which has limited the free expression of drug instructions to some extent. The Provisions for Drug Registration emphasizes the consistency in safety, efficacy and quality of generic drugs and original drugs, but it does not specify the consistency and judgment standards of the instructions. It is difficult for generic drug that is consistent with the original drug to avoid different or basically inconsistentinstructions from the original drug. This can easily lead to disputes over drug instruction infringement.

\section{ANALYSIS OF THE PROPERTY OF DRUG INSTRUCTIONS}

\section{A. The Drug Instructions Are Not Administrative Documents}

First of all, from the perspective of the documenting entity, the drug instruction is not directly formulated by the government department. China's Provisions for Drug Registration clearly stipulates that the Food and Drug Administration has the right to review and approve drug instructions, but it is also only a passive audit, and it cannot actively formulate drug instructions. [1]

Secondly, administrative documents are formulated for government organs to administer and conduct official activities according to law. The drug instruction is only used to inform the user of the specific information of a certain type of drug. It does not have a role in guiding the administrative and official activities of the country's functional departments, but only provide patients with a guide to use drugs.

Besides, the formulation and modification of drug instructions have to be performed by the informant, and the Food and Drug Administration is always in the position of guiding norms. Although the Food and Drug Administration has many mandatory specifications for drug instructions, in the final analysis, the Food and Drug Administrationcannot independently revise and formulate drug instructions. If the content of the instruction does not violate laws and regulations, the Food and Drug Administration has no right to intervene in the formulation and revision of drug instructions.

Finally, if the drug instruction does not conform to laws and regulations, or misleads users to misuse the drug, which causes adverse consequences, the legal consequences are borne by the drug producers, and the Drug Administrationdoes not assume any responsibility, for it only carries out formal review of drug instruction.

\section{B. The Drug Instruction Is the Object of Copyright Protection - Work}

China's Implementing Regulations of the Copyright Law stipulates that works protected by copyright should have originality, a certain degree of objective expression, and reproducibility in addition to being an intellectual work. At the same time, it must be created in the fields of literature, art and science. Drug instruction is created on the basis of a large number of medical experiments, which is undoubtedly an intellectual labor achievement, but if it wants to be protected by the copyright law, these four conditions are indispensable. [2]

1) Originality: There is no clear standard on originality in our country's laws, but these several viewpoints are generally accepted: only the uniqueness of the expression of the work is required; the originality has nothing to do with the quality of the work; different works have different requirements for originality; the possibility of "similar works"is recognized. [3]

The essence of drug instruction is an instruction of a certain product, whose unique textual expression and content of scientific research achievements reflect the originality. First of all, the data in clinical trials involved in the instruction is an intellectual labor achievement itself; secondly, the author should illustrate the effects and characteristics of the drug as concisely as possible in order to arouse the attention of the user on the basis of not violating the relevant laws and regulations. This all reflects the wisdom of the author and meets the definition of originality standards. [4] 
2) Ability to reproduce with a tangible carrier: The copyright law stipulates that works can be reproduced with tangible material carriers, such as the words and symbols that people can see as well as sounds that people can hear. The drug instruction clearly meets this requirement.

3) Reproducible: Completing the creation of a work does not mean that its value has been achieved, and its true value can only be achieved when the work is spread and used. So many countries take reproducibility as an important criterion to judge a work. Drug instructions appear with drugs and can obviously be reproduced in some tangible form.

4) Creation in the field of natural science: According to the provisions of China's Copyright Law, the scope of works is the fields of literature, art and science. Drug instruction is a detailed explanation and description of drug information from the perspective of content. Its creation requires a large number of experiments on animals and humans, and the creation is completed by summarizing the data. Therefore, the material pf drug instructionis derived from natural sciences. Therefore, drug instructions can be said to becreated in the field of natural sciences.

In summary, drug instruction is in line with all the conditions that a work in the sense of the Copyright Law should have, and it should be protected by China's Copyright Law.

\section{Obstacles to the Copyright PRotection of DRUG INSTRUCTION}

\section{A. Proposal of the Question}

1) Different verdictsin the same case: In the case that Wellman Pharmaceutical Co., Ltd. sued Erye Pharmaceutical Co. for infringement, Wellman Pharmaceutical Co., Ltd. believed that Erye Company's "Piperacillin Sodium and Sulbactam Sodium for Injection" instruction is basically the same as their "Piperacillin Sodium and Tazobactam Sodium for Injection (2:1)"instruction and its copyright isinfringed. The court of first instance held that the instruction of "Piperacillin Sodium and Tazobactam Sodium for Injection (2:1)" was original and enjoyed copyright, and Erye Company was sentenced to immediately stop the infringement and make economic compensation to Wellman.[5] Erye Company subsequently filed an appeal and the second-instance ruling upheld the original verdict, saying that although the formulation of the drug instruction was approved by the government department, it was not in the scope of administrative documents, not to mention some data and experimental procedures involved in the drug instruction were all Wellman Company's intellectual achievements and were original, and that the Copyright Law should protect such product instructions. [6]

In the case that Wellman Pharmaceutical Co., Ltd. sued Foshan Hongxing Pharmaceutical Co., Ltd. for copyright infringement, the defendant had been selling the "Piperacillin Sodium and Sulbactam Sodium for Injection" of Erye, and had been using the "Instruction of Piperacillin Sodium and
Sulbactam Sodium for Injection". The victory in suing Erye before Pharmaceutical Company convinced Wellman and it sued the Hongxing Pharmaceutical Co., Ltd. for the infringement of the drug instruction's copyright. The court of first instance supported the appeal of Wellman, and demanded that the infringement should be stopped immediately and the liability should be compensated.[7]However, the secondinstance judgment was in contrast to the first instance. Foshan Intermediate People's Court held that the drug instruction was essentially different from the work and could not be classified as a work. Therefore, it should not be protected by the Copyright Law. [8]

2) Focus of the dispute: Through these two cases with very different verdicts, we can see that the focus is whether the drug instruction is a work and whether it should be protected by the copyright law. Those who agree with it believe that drug instruction is a result of people's scientific research, and has certain originality and deserves protection. Those who disagree with it believe that the formulation process of the drug instructionis subject to rigorous examination by the drug regulatory authority, with strict specifications in format and content. If it fails, the relevant administrative department will order it to be revised. The right of revision is a personal right of the author, and whether to revise, how to revise, and when to revise should be decided by the author himself/herself, rather than follow administrative orders. This has become an obstacle for authors of drug instructions to exercise rights. In addition, the contents involved in the drug instructions include experimental data of some drugs, while methods of use and function introductions are not in the protection scope the copyright law.

\section{B. Analysis of the Reasons for Disputes over the Copyright of Drug Instructions}

1) There is no clear standard of the "originality": The definition of works is stipulated in Article II of the Implementing Regulations of the Copyright Law. However, this article only clarifies that works should be original and does not strictly define and explain originality.

Originality is a substantive component that constitutes works on the level of copyright law. Different countries have different legal and cultural backgrounds, and their respective legislative purposes are not uniform. The "statement of commercial copyright" is the philosophical basis for the formulation of laws in countries of the Anglo-American legal system. They use the definition "Copyright", and the purpose of legislation is to protect the author's interests from a certain work, thereby stimulating people to invest more in the creation of works to ensure the smooth dissemination of these new works. The threshold for originality of works is particularly low. The countries of civil law system believe that the value of personality should be the philosophical basis for the formulation of laws, and it 0must be based on the protection of the author's personal interests. They use the definition "author right", in which "Author" is also the author's own rights and interests. It can be seen that the purpose of the Copyright Law is to motivate people to exert their intelligence and intellectual creativity. The civil law system is much stricter than the 
Anglo-American Legal Systemin terms of originality. However, with the exchange of cultures in different countries, the two major legal systems also draw lessons from each other in the determination of originality. The Anglo-American Legal Systemis constantly improving its own requirements for originality. At the same time, the civil law system also flexibly reduced its own criteria.

2) Law's restriction on drug instructions:As a special commodity, the instruction of drugis an important reference for guiding people to use drugs rationally. If the content is not accurate or scientific, it will pose a serious threat to the health and life of users. Therefore, all countries in the world have formulated corresponding laws to standardize the contents and format of drug instructions, strictly require the formulation process and revision methods of drug instructions, and form a legal restriction on drug instructions.

a) The law strictly regulates the content and format of drug instructions: Drug instructions play a decisive role in ensuring people's drug safety and right to know about medical products. Therefore, all countries have strict regulations on the content and format of drug instructions.

The United States has strict laws and regulations on drug administration and also has a complete set of management system for drug instructions. The US National Food and Drug Administration (FDA) categorizes prescription and nonprescription drug instructions for management. For prescription drugs, all the contents of the instruction are required to be clarifiedaccurately and specifically, with professional and comprehensive expression. But the requirements for non-prescription drugs are looser, because consumers can purchase them directly. Therefore, the FDA does not specify that non-prescription drugsmust be accompanied by instructions, but just requires explicit instructions for drug use on the packaging label. At the same time, the FDA also classifies drugs into original drugs and generic drugs, and stipulates that the label of generic drugs must be the same as that of original drugs, and the content and format of the label should be updated at any time. The EU's regulations for generic drug instructions are different from those in the United States, which require that instructions do not have to refer to the original drug. However, the requirements on instruction content and format are also very strict and specific.

Article 54 of China's Pharmaceutical Administration Lawstipulates that label must be printed on or affixed to the packaging and the instruction must also be attached in accordance with regulations. At the same time, the content of the label or the instruction must include the generic name of the drug, the specific chemical composition, the specifications of the drug, the manufacturer's specific information, etc., and also indicate the applicable population, the usage and dosage of the drug. At the same time, it is also requires that the items indicated in the instruction must be complete and normative, and information about the drug must be accurate, clear and detailed. There are also strict requirements on the format and content of drug instructions in the Specifications for Instructions of Chemicals and Therapeutic Biological Products. b) The law strictly supervises the formation procedure of drug instructions

- The formation of drug instructions should be completed under the supervision of the law

The drug instruction is closely related to the health of the public. All drug instructions in various countries must be reviewed and approved by the national drug regulatory department before they can be released to the public. The FDA is responsible for reviewing the US local drug instructions and labels. Manufacturers draft the instructions and attach the specific experimental data involving the contents of each item to the FDA for review. The FDA and manufacturers discuss the revision and finalize the draft. In our country, the applicant for registration of the drug first needs to submit an application to the relevant department for the contents of the drug instruction. The relevant technical review unit will perform the verification according to the application materials and relevant audit opinions. The relevant department will conduct a comprehensive inspection on the applied drug instruction after the review is completed and the result of the consent is obtained.

In addition, the completion date of the drug instruction is different from the writing date. The instruction can be considered to be formed only when the drug regulatory department approves it, which is also different from the automatic acquisition of copyright. In addition, drug instruction, as a subsidiary document of the drug, does not have any meaning or its own independent value if it is separated from the drug itself, while works, in general, exist for the purpose of expressing certain ideas and have their own independent value and meaning of existence. This is also one of the important reasons why the drug instruction is excluded out of the scope of works by people.

- The revision of drug instruction should be completed under the supervision of the law

According to the law, the revision of drug instruction by enterprises is not arbitrary. In many cases, it is based on the mandatory requirements of the drug regulatory department. This is also a big difference between the drug instruction and the characteristics of general works. The general worksare revised according to the creator's own wishes, which is absolutely free and will not be restricted and controlled by the relevant departments.

\section{SugGeStions FOR RESOLVING OBSTACLES TO COPYRIGHT PROTECTION OF DRUG INSTRUCTIONS}

\section{A. Adopt a "Moderately Creative Height" Standard}

We should consider the essence of copyright disputes on drug instructions and the fundamental purpose of the pharmaceutical industry to serve public health, comply with China's pharmaceutical industry, and make full use of the opportunity brought about by the expiry of patented drugs to China's generic drug development at present, focuson introducing technology and encourage independent innovation, protect imitation and innovate in imitation. We can adopt the "moderately creative height" standard [9] to confirm whether 
the drug instruction is a work. This standard is different from the "intellectual creation" standard of countries of civil law system, and it is also different from the "independent completion" standard of the countries of the Anglo-American legal system, as long as the work is completed by the author independently rather than plagiarizing or imitating the works of others and can reflect some personalized features

\section{B. Follow the Principle of "Overall Recognition and Partial Protection"}

The originality of different components should be explained based on "whether or not the expressioncontents and forms are unique", and the original parts such as pharmacokinetics should be protected, while those nonoriginal parts, such as drug specifications, are not protected.[10] In addition to the integration theory of the United States, in the case Baker v.Selden, the Federal Supreme Court of the United States considered the practicality of original works through integration theory. The judge of the case thought that the craft could not be used in addition to adopting methods and diagrams used in this book to describe a certain craft, or adopting similar methods and icons, and the methods and icons to describe the craft should belong to the public domain and should not be protected by copyright as essential components of the craft. [11]]After excluding the non-original and removing the part of the public domain, the remaining original part should be protected by copyright.

\section{Balance Public Interests}

Drugs are used to cure and save people, however, at present, the circulation can not benefit all people. If drug instructions are protected by copyright, generic drugs will be greatly restricted. For a country like China that is dominated by generic drugs, it may hinder the development of the pharmaceutical industry. China's relevant laws and regulations impose strict restrictions on the content and format of drug instructions. If drug registration applicants or $R \& D$ producers change the format of drug instructions, they will not be able to pass the review of the Food and Drug Administration. If they do not revise the format, they will be suspected of infringing on copyright, which will block the circulation of cheap generic drugs. In order to avoid infringement, generic drug manufacturers can only try their best to change their expressions, which may obscure simple explanations and is not conducive to the purchase and use by patients. In addition, drug instructions are scientific information based on summarizing test results. If generic drugs are required to have different drug instructions, generic drug manufacturers will be forced to spend huge sums of money to carry out unnecessary repetitive experiments, which will greatly waste resources and time, and increases the burden.

In summary, analyzing from the perspectives of legal and academic principles, copyright protection can be applied to drug instructions, but it should make appropriate concessions, give up some of the rights to adapt to the objective needs of generic drug development. We can draw lessons from the legislation of the United States and Taiwan, China. Even if the instructions of generic drugs and the original research are consistent, based on the parties' trust in the laws and regulations and the use of reference drugs against the relevant policies formulated by the CFDA, it is not judged as infringement of copyright.

\section{CONCLUSION}

Drug instruction has a certain degree of originality, and meets the conditions of works, so it should be protected by copyright law. However, we should combine with the integration theory, recognize as whole and protect partially, and should pay attention to the protection of public interestsat the same time. In related legislation and judicial activities, our country should draw on the practices of the United States and Taiwan to rationally and legally resolve disputes on drug instructions and safeguard justice and equity of laws.

\section{REFERENCES}

[1] Ding Wenyan: "Causes of Copyright Issues in Drug Instructions and Resolutions", Journal of Law Application, No. 6, 2012.

[2] Chen Xiaochen: "DoDrugs Belong to Works in the Sense of Copyright Law?", People's Judicature, No. 20, 2012.

[3] Feng Xiaoqing: Copyright Law, Beijing: Legal Press, 2010, p. 49.

[4] Li Mingde, Xu Chao: Copyright Law, Beijing: Legal Press, 2003, p.27.

[5] Changsha Yuelu District People's Court (2009) Yue Min Chu Zi No. 02469 Civil Judgment.

[6] Changsha Intermediate People's Court (2010), Chang Zhong Min San Zhong Zi No. 0437 Civil Judgment.

[7] Chancheng District People's Court of Foshan (2012) Fo Cheng Fa Zh Min Chu Zi No. 279 Civil Judgment.

[8] Foshan Intermediate People's Court (2013) Fo Zhong Fa Min San Zhong Zi No. 14.

[9] Intellectual Property Trial of Beijing No. 1 Intermediate People's Court: Overview of Intellectual Property Trial, Intellectual Property Publishing House, 2008 edition, p.9.

[10] Drug Instructions Shall be Protected by the Copyright Law - Interview with Prof. Wang Guangji, Vice President of China Pharmaceutical University, China Pharmaceutical News, 2012/July/23/003th edition

[11] Zhao Li: "Analysis of Several Legal Issues in the Case of Australia's 'Sanofi-Aventis' Company", Journal of Xi'an University of Electronic Science and Technology, No. 2, March 2013. 